\title{
Tangence
}

\section{Les récits du Canadien}

Politique, fiction et nation

\section{Micheline Cambron}

Numéro 63, juin 2000

Fictions et politique

URI : https://id.erudit.org/iderudit/008185ar

DOI : https://doi.org/10.7202/008185ar

Aller au sommaire du numéro

Éditeur(s)

Presses de l'Université du Québec

ISSN

0226-9554 (imprimé)

1710-0305 (numérique)

Découvrir la revue

Citer cet article

Cambron, M. (2000). Les récits du Canadien : politique, fiction et nation.

Tangence, (63), 109-134. https://doi.org/10.7202/008185ar d'utilisation que vous pouvez consulter en ligne.

https://apropos.erudit.org/fr/usagers/politique-dutilisation/ 


\section{Les récits du Canadien. Politique, fiction et nation}

\section{Micheline Cambron, Université de Montréal}

Le journal Le Canadien peut être considéré comme le lieu de naissance de la nation canadienne. Certes, le mot "canadien" était déjà usité ${ }^{1}$, mais les flottements de l'ethnonyme trouvent là, en 1806, un ancrage nouveau dans un titre, celui du journal (rappelons que le titre du premier journal écossais, sera lui aussi un ethnonyme The Scotman), et dans une nouvelle articulation du politique, concomitante à l'émergence d'un espace public propre. Ce journal naît, rappelons-le, en réaction aux textes du Québec Mercury, perçus comme diffamatoires. Il s'agit de se servir de la liberté de la presse pour corriger les "insinuations" parues dans le Mercury afin de "dissiper les préjugés qu'entretient ce parti envieux dans l'esprit d'un nombre des anciens sujets de Sa Majesté, avec qui ils ont à vivre unis dans ce pays, [...] et peut-être dans celui même de Sa Majesté". D'emblée, Le Canadien se situe donc dans un espace polémique. Son projet est fort éloigné de celui de simple relais de l'information explicitement revendiqué par $L a$ Gazette de Québec en 1774, là où l'on désire rendre compte de tous les événements, européens, américains ou britanniques en "autant qu'on les jugera dignes de l'attention du lecteur comme matière d'amusement, ou qu'elles puissent être utiles au Public en qualité d'habitants d'une colonie Angloise", laissant place aux productions locales au moment où "la rigueur des Hivers suspend l'arrivée des navires", dans une sorte de parenthèse du politique donc. D'ailleurs, "PARTY PREDJudice, or Private SCANDAl, will never find a place in this Paper". Certes, la lecture de la Gazette de Québec montre que ces résolutions sont parfois négligées ${ }^{2}$,

1. Voir Gervais Carpin, Histoire d'un mot. L'ethnonyme Canadien de 1553 à 1691, Sillery, Septentrion, 1995.

2. Il y a quelques polémiques plus animées, mais l'effacement de l'espace public national est net pour de longues périodes. Par exemple, la lecture du journal entre décembre 1799 et février 1800 montre bien l'indifférence à l'espace national, qui n'est guère lisible qu'à travers la représentation du commerce (les annonces) et les interpolations de dates rendues nécessaires par la lenteur que mettent les informations à traverser l'Atlantique. Plus rarement, 
mais son prospectus ne pose pas d'emblée cette publication dans un espace public "national", sinon par le biais d'une vague définition géopolitique - la "colonie angloise" — et météorologique - la "rigueur des Hivers". L'espace discursif du Canadien ne se confond pas non plus avec le phantasme d'une société littéraire tel que Bernard Andrès a pu le déceler dans la première Gazette de Montréal (1777-1779) ${ }^{3}$. Le prospectus du Canadien n'évoque aucunement la littérature, non plus que le divertissement des lecteurs: Le Canadien se veut ouvertement un journal d'opinion qui témoigne du politique tel que les lignes de parti le construisent.

Cependant, ce journal, comme toutes les feuilles qui lui sont contemporaines, demeure profondément littéraire, c'est-à-dire inscrit dans une tradition de lecture littéraire. Cela tient, si l'on en croit Habermas, à ce qu'en cette période d'émergence de l'espace public, la littérature offre ses formes à un substrat politique, livré à un public qui le juge, la Presse permettant la cohabitation de l'espace privé et de l'espace public grâce au "recyclage" de modalités de lecture ${ }^{4}$. Graduellement, de l'exercice privé du jugement, on passe à un exercice public, l'espace public prend "forme" jusqu'à gagner en autonomie - ce que confirme l'hégémonie alors acquise par la publicité —, et le journal, plurigénérique et polyphonique, crée l'illusion de l'unicité de l'opinion publique. De ce point de vue, le journal, objet qui condense en un espace clos le disparate infiniment expansible de l'espace public, est sans doute à l'origine de l'idée de nation, il en constitue la fic-

la publication d'édits gouvernementaux permet de soupçonner un horizon strictement local.

3. Voir "Le fantasme du champ littéraire dans la Gazette de Montréal (17781779)", Etudes françaises, vol. 36, n 3, à paraître.

4. Celles-ci passent de la sphère privée à la sphère publique. L'espace public. Archéologie de la publicité comme dimension constitutive de la société bourgeoise, Paris, Payot 1992 [1962]. Il y a apparemment contradiction entre cette position et celle de Marshall McLuhan, selon lequel "le médium est le message". Mais il est ici question moins de la tension entre forme et contenu qu'entre contenu et modalités de lecture durant la période d'émergence de la presse (fin $\mathrm{XVII}^{\mathrm{e}}$, début $\mathrm{XVIII}^{\mathrm{e}}$ siècle), lesquelles modalités sont par ailleurs graduellement infléchies par les nouveaux médias. Notons par ailleurs que l'emploi que fait Habermas du mot littéraire est le plus souvent associé à la matérialité de l'écriture - dans un sens assez semblable au literacy anglais. Cependant, pour cette période d'émergence, c'est le littéraire dans sa forme qui intéresse Habermas: roman sentimental ou épistolaire, dont le Pamela de Richardson serait un exemple paradigmatique; critique d'art, dont le modèle serait les Salons de Diderot. 
tion fondatrice et utopisante. Je poserai cette hypothèse à l'horizon d'une lecture du journal Le Canadien, cherchant à y découvrir, dans et à travers les textes qui le composent, du récit, c'est-àdire une mise forme empruntée à l'ordre de la fiction et organisant le substrat politique. Cette lecture nous permettra de saisir dans le vif du discours les paradigmes narratifs originels à l'œuvre et, en bout de ligne, de revenir brièvement sur les relations complexes qui unissent la fiction, le politique et l'identité narrative dans le discours culturel de la première moitié du $\mathrm{XIX}^{\mathrm{e}}$ siècle canadien.

J'examinerai plus particulièrement le prospectus, le premier numéro et l'Etrenne du Gazettier, tout en circulant dans les neufs premiers numéros parus, c'est-à-dire ceux du 22 novembre 1806 (vol. 1, $\mathrm{n}^{\circ}$ 1) au 17 janvier 1807 (vol. 1, no 9), lesquels numéros comportent en condensé les thèmes, motifs et traits argumentatifs marquant la première période du journal, qui s'achève abruptement au moment de la saisie des presses et de l'emprisonnement des propriétaires par le régime Craig ${ }^{5}$. Je resserrerai mon analyse autour de deux éléments discursifs centraux, l'image de la Révolution et le statut de l'archive, à partir desquels nous serons à même de dégager une conception du temps historique et de la raison dont nous verrons qu'elle joue un rôle déterminant dans la mise en forme des récits réflexifs qui donnent sens à toute l'aventure du Canadien.

\section{Les récits du Prospectus}

Une première version du récit du journal ${ }^{6}$ nous est livrée sans fard dans le Prospectus qui en annonce la parution. Dès les premières phrases, le sujet énonciateur se présente comme partie d'un "nous" uni par un pacte, celui de la constitution; à l'intérieur de ce "nous", un petit groupe, "des personnes qui aiment leur pays et leur Gouvernement", manifeste son regret que "le trésor rare que nous possédons dans notre constitution, demeure si

5. Le 1810. La lutte du journal avec Craig est fort bien retracée dans Jean-Pierre Wallot, Le Bas-Canada sous l'administration Craig, thèse soutenue à l'Université de Montréal, 1965, p. 372 et suivantes.

6. Pour une définition de ce concept, voir Micheline Cambron (dir.), Le Journal Le Canadien. Littérature, espace public et utopie, 1836-1845, Montréal, Fides, 1999, p. 43-64. 
longtemps caché, faute de l'usage de la liberté de la presse". En effet, "le droit qu'a un peuple Anglois, sous une telle constitution, d'exprimer librement ses sentiments sur tous les actes publics de son Gouvernement, est ce qui en fait le principal ressort". Il n'y a rien d'original dans cette profession de foi héritée des Lumières, auxquelles ces premières lignes empruntent à la foi une topique — la liberté de la presse — et une image cardinale — "l'office [de la liberté de la presse] est de répandre la lumière sur toutes ses parties". Cependant l'entrelacement complexe des sujets grammaticaux mérite qu'on s'y arrête. Si l'existence d'une petite phalange éclairée, avide de liberté, au sein de la communauté du "nous" paraît aller de soi, l'article indéfini qui détermine "peuple Anglois", et l'ambiguité du possessif ${ }^{7}$ - le peuple possède "son Gouvernement " tout autant que l'inverse - suggèrent un déplacement significatif. L'ambivalence du possessif laisse planer un doute quant à la notion de sujet, puisque le sujet devient alors à la fois celui qui est soumis, lié à un gouvernement par un lien de sujétion et celui qui exerce ses droits de sujet souverain. Ainsi l'aire d'action du sujet demeure-t-elle indéterminée. De son côté, l'usage de l'article indéterminé pour parler du "peuple anglois" force le lecteur à induire qu'il existe non pas un, mais des peuples Anglois et qu'un Anglois peut donc être défini de deux façons, soit par son origine - auquel cas l'Anglois et le Canadien sont distincts ${ }^{8}$ - , soit par la constitution qui fonde le "nous" "notre constitution". Le Canadien est donc à la fois l'Autre et le Même de l'Anglais, son appartenance à un même "peuple" " est postulée. Or, dans cette perspective, le Canadien entretient avec "l'exécution exacte des lois" une relation qui ne saurait être maintenue intacte, selon les termes du texte, que si le peuple peut exercer son "pouvoir censorial" grâce à la liberté de la presse. Par ce pouvoir, en effet, le peuple et le Gouvernement deviennent des instances sinon égales, du moins équipollentes, puisque "la liberté de presse, en faisant connoître le peuple à celui qui gouverne, fait connoître au peuple l'excellence de son gouverne-

\footnotetext{
7. Grammaticalement, il y a indétermination du lien de possession, c'est l'usage qui détermine l'orientation vectorielle qui va du "possédant" au "possédé".

8. Comme dans le segment de phrase: "en quoi consiste la liberté d'un Anglois, qui est à présent celle d'un Canadien".

9. Faut-il préciser que la célèbre locution des deux "peuples fondateurs" est ici totalement anachronique, sinon lorsqu'on pense aux peuples continentaux à l'origine du peuple nouveau lié par la constitution?
} 
ment", ce qui explique qu'«il le regarde comme à lui". La constitution d'Angleterre est ainsi "propre à faire le bonheur des peuples qui sont sous sa protection". Le "nous constitutionnel", celui du "peuple", est à la fois uni, parce que la "communication continuelle, entretenue par la liberté de la presse, entre le Gouvernement et le Peuple unit étroitement l'un à l'autre de sentimens, et forme ce tout si désiré, mais si rarement obtenu dans la formation des États", et diffracté selon les pays placés "sous [la] protection" de la constitution. Si le "nous" peut être partagé suivant une ligne historique qui permet de distinguer entre anciens sujets et nouveaux sujets, les Canadiens étant "les plus nouveaux", l'union dans l'ordre constitutionnel caractérise le présent. D'ailleurs, toute la première partie du texte, qui est une défense et illustration de la liberté de presse et de la constitution anglaise, se conjugue au présent, un présent qui est à la fois celui ponctuel de l'énonciation et celui, éternel, des principes qui régissent l'économie politique. Dans ce présent, où règne le bonheur constitutionnel et d'où la révolution est exclue («il est impossible que de pareils inconvéniens aient lieu"), la presse constitue un espace de représentation dont l'existence "efface les divisions" entre les "parties du peuple", car le préjugé "est lui-même entretenu par l'isolement et le défaut de communication". La connaissance mutuelle rendue possible par la "communication continuelle" est donc présentée comme un idéal accessible dans l'absolu de la loi.

La seconde partie du texte rompt cependant avec cette représentation idyllique. Introduite par un "mais", la suite du texte expose les nécessités de l'exercice de la liberté de presse, qui "asservi à un parti [...] ne serviroit qu'à entretenir des divisions odieuses, à entretenir d'un côté des préjugés injustes, et à faire sentir profondément à l'autre côté, l'injustice de la calomnie, sans lui laisser les moyens de la repousser". Selon le journal, il semble bien qu'au Canada l'exercice de la liberté de presse ait créé de telles divisions: le texte nous apprend que le "nous" idyllique a été nié dans un "papier publié en Anglois". Le passé fait ainsi irruption: "Il n'y a pas bien longtemps qu'on les [les Canadiens] a vu flétris par de noires insinuations". On remarquera au passage qu'il ne s'agit pas d'un passé lointain - la menace faite au "nous" ne renvoie pas à la Conquête, par exemple ${ }^{10}$-, mais

10. Philippe Reid (Représentations idéologiques et société globale. Le journal Le Canadien (1806-1842), thèse soutenue à l'Université Laval, 1979) a déjà noté 
114

d'un passé proche, qu'une action immédiate peut neutraliser: la liberté de presse permet aux Canadiens de "venger la loyauté de leur caractère", d'ailleurs "ils ont intérêt de dissiper les préjugés qu'entretient ce parti envieux dans l'esprit d'un bon nombre des anciens sujets de sa Majesté avec qui ils ont à vivre unis dans ce pays". Le présent en est donc un de défense contre "les mauvaises impressions " et de rectification de la représentation. Il met en scène, les Canadiens, nouveaux sujets, les Anglais, anciens sujets, et le parti envieux qui, par l'intermédiaire d'un "papier Anglois" (il s'agit du Quebec Mercury, qui n'est pas nommé), exprime "déloyauté", "bassesse", "envie" et "sème la division entre des concitoyens qui ont à vivre en frères". La distinction entre anciens et nouveaux sujets est ainsi présentée comme un événement advenu, lié à la mauvaise foi d'un "ils" qui cherche à diviser le "nous" de ceux qui ont à vivre dans l'harmonie de la constitution britannique. Il s'agit d'une division liée à l'ordre de la représentation plutôt qu'à l'ordre historique, ce qui rejoint la profession de foi de la première partie du texte, qui dissolvait la dimension historique de la nation dans l'équanimité créée par la perfection de la constitution, fondée dans l'éternel présent d'une représentation commune, source d'affection, de paix et de stabilité politique. Le dernier paragraphe du Prospectus nous ramène d'ailleurs à cette harmonie, car si les Canadiens soutiennent la liberté de presse, comme on les y invite, on prédit que les divisions cesseront puisque "on les connoîtra tels qu'ils sont". Les préjugés étant dissipés, "Fiat Justitia ruat Coelum", selon le "motto du papier": que la justice renverse le ciel (ou l'atmosphère ou le climat de la contrée).

Dès l'origine, nous sommes donc en face de deux récits distincts, dont l'un surplombe l'autre à hauteur d'éternité. Il y a le récit idéal, celui que porte en germe la constitution, dans lequel un sujet collectif uni, le "nous" qui regroupe à la fois les anciens et les nouveaux sujets, vit dans l'harmonie d'une représentation qui a dissout tout préjugé, et promet un présent éternel, celui de la Loi. Ce récit, qui comporte à l'évidence une forte composante utopique, ne porte d'autre action que celle de la loi elle-même,

la rareté des renvois à la période d'avant la Conquête, laquelle est présentée non pas comme un âge d'or, mais comme obscurantiste (p. 122). Pour un regard synthétique sur la première série du journal et de substantielles citations, on peut se reporter aux p. 75-138. 
infiniment reconduite, puisque toute révolution est "impossible". Nous sommes dans une structure narrative d'où les conflits sont exclus, évacués qu'ils sont par la représentation qui soude la communauté. Ce récit, qui est raconté en creux et qui constitue une refiguration consciente de la part des rédacteurs du journal, est projeté dans le futur, les grenouillages du passé récent et du présent ponctuel n'étant qu'un événement accidentel et somme toute accessoire, une sorte de déni improbable de la structure narrative proposée comme relevant de l'ordre de la Loi. Remarquons également que l'espace de ce récit est étrangement expansible puisque le "nous" rassemble de fait diverses communautés reliées entre elles par la constitution anglaise et échappant ainsi aux maux qui accompagnent tout gouvernement despotique sujet "à de si terrible révolutions". L'espace n'est donc plus circonscrit par des frontières nationales, mais bien juridiques.

Cependant, les événements du passé récent et du présent ponctuel composent un autre récit, autonome, répondant à d'autres paradigmes. Le sujet y est diffracté en trois instances subjectales: deux fractions du "nous" du premier récit, un "ils" et un "nous", créées par le "on" du parti "envieux", lequel représente la troisième instance. La temporalité, loin d'être réduite à un présent éternel et à ses prolongements naturels dans le futur, est vectorielle, tendue entre le passé de la loyauté bafouée, le présent de l'honneur à venger et le futur de l'existence du journal que promet le Prospectus. Quant à l'espace, il est singulièrement rétréci puisqu'il est question de "concitoyens", Canadiens et "anciens sujets", "qui ont à vivre unis dans ce pays", dans un espace bien délimité donc. Ce second récit, que le journal à venir se propose de désamorcer et de faire disparaître est mutatis mutandis celui du Quebec Mercury, le "on", non identifié explicitement, qui distingue entre un "nous" et un "ils" issus des différences de langue, selon le Prospectus. Certes, la topique du récit est inversée: ce sont les Canadiens qui sont déloyaux pour le Mercury, alors que c'est le "on" du Mercury qui est déloyal pour Le Canadien Mais pour l'essentiel, le récit est semblablement structuré. D'une certaine manière, Le Canadien propose deux récits placés en concurrence et suggère de faire triompher le premier.

Avant d'entrer dans le vif de la discussion qui s'amorcera dès les premières livraisons du journal, arrêtons-nous brièvement sur le récit privilégié par Le Canadien. Celui-ci tire sa cohérence d'ellipses troublantes. Ainsi, l'impossibilité de la révolution sous 
la constitution anglaise qui se trouve postulée occulte la Révolution d'Angleterre (1688) et la Révolution américaine et renvoie $a$ contrario à une seule et même logique toutes les autres révolutions européennes. De plus, la césure de la Conquête est quasi passée sous silence - n'en reste que la trace que constitue la distinction entre anciens et nouveaux sujets - et cette division est réduite à une question de langue. Enfin, la religion ne prend aucune part dans le discours:

En un mot par la liberté de la presse la constitution d'Angleterre est celle dont on peut dire qu'elle ne craint ni l'œil ni l'observation du sujet

Placé en plein centre du texte, l'œil du sujet remplace ici l'œil de Dieu.

Ces deux ellipses, celle de la réalité des révolutions sous la constitution anglaise et celle de Dieu, suggèrent une conception paradoxale de l'histoire. En effet, l'éternité en laquelle est figée la perfection constitutionnelle paraît renvoyer à la conception providentialiste de l'histoire qui a trouvé sa forme achevée dans les théories absolutistes de Bossuet, de Fénelon et les représentations plus laïques de la société mises en forme par John Locke ${ }^{11}$. Il faut rappeler que la conception providentialiste de l'histoire est très présente dans le Canada de l'époque: le discours où $\mathrm{M}^{\mathrm{gr}}$ Plessis présente la Conquête comme un geste divin protégeant la colonie des affres de la Révolution française date de 1798 et cette interprétation sera reprise et explicitée par des laïcs, dont Louis Plamondon et Denis-Benjamin Morin en $1809^{12}$. Cependant, le rôle prééminent accordé à la liberté de la presse, la

11. Pour une analyse tout à fait éclairante des enjeux philosophiques, historiographiques et politiques de ces discussions, voir Jean Goulemot, Le règne de l'histoire. Discours historiques et révolutions, XVII ${ }^{e}-X V I I I^{e}$ siècle, Paris, Albin Michel, 1996, particulièrement les chapitres VI et VII.

12. Voir à ce sujet David M. Hayne, "Conquête providentielle et Révolution diabolique. Une constante de la littérature québécoise du XIXe siècle ", La Révolution française au Canada français, Ottawa, Actexpress, Presses de l'Université d'Ottawa, 1991, p. 321-337. Cette idée demeurera présente tout au long du XIX ${ }^{e}$ siècle. On en trouve la trace exacerbée dans le célèbre sermon prononcé le 23 juin 1902 par M ${ }^{\text {gr }}$ Louis-Adolphe Paquet, La vocation de la race française en Amérique (reproduit dans Anthologie de la littérature québécoise, sous la direction de Gilles Marcotte, tome 1, Montréal, l'Hexagone, 1994, p. 789-797). Pour une lecture des avatars littéraires de cette conception de l'histoire, voir Réjean Beaudoin, Messianisme littéraire au Canada français, 1850-1890, thèse soutenue à l'Université McGill, 1987. 
nécessité de la temporisation du pouvoir monarchique par le "pouvoir censorial" du citoyen ("toute la nation devient pour ainsi dire le conseiller privé du Gouvernement"), la conviction que la représentation de l'état ne peut être restreinte à celle du pouvoir mais tient plutôt dans la coexistence de la représentation de toutes les parties du peuple et du Gouvernement, renvoient à une vision constructiviste de l'histoire, laquelle se ferait dans l'interaction de divers agents plutôt que d'être inscrite à l'avance dans le plan divin.

Il faudrait ici pointer du doigt les signes d'une lecture attentive des philosophes politiques du $\mathrm{XVIII}^{\mathrm{e}}$ siècle: outre une évidente admiration pour la constitution anglaise, sans doute héritée de Montesquieu, et une définition du préjugé injuste classique à l'époque des Lumières (il s'agit de fictions dont la connaissance et la réflexion nous libèrent ${ }^{13}$ ), on trouve un démarquage assez évident du texte de Jaucourt sur la Presse, paru dans l'Encyclopé$d i e^{14}$ et cette idée phare: celle du bonheur des peuples que "tous les gouvernements doivent avoir [pour] but ${ }^{15}$ ". La configuration du politique qui en découle est toute laïque. Le Prospectus fait donc cohabiter une philosophie de l'histoire et une conception du politique qui sont, en apparence du moins, antithétiques. Le paradoxe peut se résoudre de deux façons. Soit le politique n'est qu'un mécanisme d'équilibration tendant à permettre le maintien d'un état d'harmonie et de stabilité pérenne et il demeure alors soumis à la lecture providentialiste, soit l'harmonie relève d'une logique législative plutôt que téléologique, le politique proposant des accommodements dans la matérialité des représentations. Entre les deux, Le Canadien ne tranche pas. Le nom même du journal témoigne de cette ambiguité: Le Canadien, "c'est celui dont l'honneur est à venger", mais le journal qui vise à le représenter cherche à inscrire cette représentation dans un ensemble plus large qui serait celui de la communauté constitutionnelle et donc, en bout de ligne, à effacer la division entre les anciens et les

13. Voir la définition à l'article "Préjugé" du Dictionnaire philosophique de Voltaire, Paris, Gallimard, coll. "Folio classique", 1994, p. 437-440.

14. Encyclopédie ou Dictionnaire raisonné des sciences des arts et des métiers, choix d'articles par Alain Pons, Paris, coll. "J'ai lu", 1963, p. 478-479.

15. Rappelons que cette idée apparaît d'abord chez Hobbes puis chez Locke avant d'être reprise par Rousseau. Robert Mauzi a longuement montré le rôle central du bonheur dans la pensée des Lumières (L'idée de bonbeur au XVIII siècle, Paris, A. Colin, 1967). 
nouveaux sujets. Cela ne l'empêche pas de vouloir maintenir le trait distinctif qu'est la langue, tout en le subordonnant à des valeurs universelles: "l'expression sincère [...] de la déloyauté, de la bassesse et de l'envie [...] déshonorent [sic] également toutes les langues ${ }^{16}$ ". Si dans l'empyrée constitutionnel, les différences s'effacent, dans la concrétude de la représentation, il faut néanmoins laver son honneur et témoigner de ses différences ${ }^{17}$. Rien d'étonnant à ce que cette structuration paradoxale soit particulièrement perceptible lorsque est mise en jeu la conception du temps, que ce soit dans sa dimension mémorielle et historique, matérialisée de manière forte dans les archives qui sont signes de continuité par delà le temps, ou dans l'irruption d'événements qui témoignent d'un Kairos aux dimensions variables, tantôt simple changement, tantôt révolution, mais, dans tous les cas, péripétie. Si, comme le dit Jacques Rancière, "il n'y a de politique que par l'interruption, la torsion première qui institue la politique comme le déploiement d'un tort ou d'un litige fondamental ${ }^{18}$ ", alors ce paradoxe désigne la naissance du politique dans le Bas-Canada.

\section{De la révolution et de quelques girouettes}

Le premier numéro (22 novembre 1806) reprend, dispersés au fil des pages, les termes du paradoxe. Les quatre pages du journal, publié sur deux colonnes, comportent deux séries de textes: les textes que nous appelleront polémiques et les "Nouvelles étrangères". Les premiers sont directement attachés à la rectification de la représentation des Canadiens: Les trois premiers "papiers [...] sont des traductions de ceux que l'éditeur du Mercury a refusé d'insérer dans la feuille du 31 mars dernier" (p. 1). Il s'agit de commentaires, voire de rectifications qui engagent clairement la polémique avec le Mercury. Ils sont suivis d'un texte qui s'attaque explicitement au Mercury et de deux entrefilets qui y sont indirectement rattachés. Les brefs récits qui sont ainsi mis en scène permettent

16. Le texte poursuit: "Ce n'est pas au langage c'est au coeur qu'il faut regarder."

17. La contradiction déjà relevée par John Hare et Jean-Pierre Wallot ("Aimant l'Angleterre et leur constitution, les Canadiens entendent demeurer des sujets britanniques et français, ce qui, pour eux, ne comporte aucune contradiction") est donc l'expression d'un paradoxe autrement plus complexe, qui met en jeu la nature même du lien social ("Le Canadien", Les imprimés dans le Bas-Canada, 1801-1810, Montréal, Presses de l'Université de Montréal, 1967).

18. La mésentente, Paris, Galilée, 1995, p. 33. 
déjà de saisir à la fois les contours d'une conception de l'histoire et certains des motifs qui seront désormais fortement tissés dans la trame des récits premiers que nous venons d'évoquer.

Le premier article est une satire qui se veut la rectification et le complément d'un texte déjà publié par le Mercury, mais dans lequel des erreurs auraient été volontairement commises, le vilain de l'histoire, montreur d'un Museum de figures de cire, n'étant pas Jean-Baptiste - représentant des Canadiens - comme l'a écrit le Mercury, mais un Yanké. Le second commente les santés "de réprobation" (p. 2) qui ont été portées lors d'un dîner rassemblant des "personnes que l'on m'avait toujours fait regarder comme des hommes loyaux et de fermes appuis de notre constitution" (p. 1), lesquelles santés auraient témoigné d'un manque de respect à l'égard de la majorité. Les personnes en question se sont défendues en prétendant qu'un tel usage était celui des Whigs, parti pourtant opposé aux convictions de ces mêmes personnes, fait malicieusement entendre le signataire, Caïus; mais cette affirmation est fausse car chez les Whigs, rappelle Caïus, " on a vu même les opposants les plus déterminés, déclarer (comme dans le cas de l'union d'Irlande) que si le Bill passoit en Loi, quoique contre leur opinion, ils feroient tous leurs efforts pour le rendre efficace" (p. 2). L'attitude anticonstitutionnelle de ceux qui ont porté les santés est, selon Caïus, signe qu'ils "désirent créer une aristocratie mercantile, le plus abominable, le plus pernicieux de tous les ordres, également préjudiciable à l'autorité de la Couronne, aux intérêts des propriétaires, et aux libertés du peuple" (p. 2). Un rappel des "usurpations" de cette classe au fil de l'histoire et une interrogation quant aux "titres" de ceux qui désirent s'ériger en un tel ordre se concluent sur l'évocation de tyrans: Tyran de Syracuse ( $\mathrm{sic}$ ), Robespierre et Catilina. "Mais notre heureuse constitution composée du Roi, des Lords et des Communes gardera, j'espère, nos descendans de l'oppression de tels personnages. Que chacun demeure dans l'état qui lui est assigné". Le troisième texte est, comme le premier, une hybridation entre commentaire politique et fiction. Il y est question du Vent de Nord-Ouest qui "devient dominant dans ce Pays", selon une remarque que l'on prête plaisamment à Volney ${ }^{19}$. Ce vent a un

19. Le signataire, Un Météorologiste, explique plaisamment que Volney est "venu ces dernières années faire des recherches sur les vents en Amérique", joli clin d'œil en direction de l'encyclopédisme du philosophe-historien. 
effet sur un "nombre considérable de Girouettes", il "vient des pays froids et porte droit vers les États-Unis". Les Girouettes sont désignées par des noms de comté et l'on comprend qu'il s'agit des parlementaires ayant appuyé des décisions profitables aux Américains. Un Météorologiste, le signataire, en appelle donc aux savants pour que le pays soit débarrassé de ce vent néfaste. Le quatrième texte est une critique de la réaction du Mercury à la parution du Prospectus, lequel soutient, dans un papier éditorial, que tous les Anglais sont visés par l'expression "envieux" employée dans le Prospectus du Canadien. Ce dernier voit dans cette insinuation une preuve de la mauvaise foi du Mercury: "L'éditeur du Mercury n'a donc pas besoin de nous avertir que les Anglais ne sont pas capables de faire le métier qu'on lui reproche, nous le savons bien; et nous croyons de plus qu'ils se passeroient bien de voir un pareil peintre [le Mercury] se mêler de travailler à leur portrait " (p. 3). Enfin, les deux entrefilets constituent des jugements lapidaires, le premier à propos des règles auxquelles doit se soumettre un magistrat ${ }^{20}$, le second sur les "successions d'idées tout à fait curieuses" que la parution du $\mathrm{Ca}$ nadien entraîne chez le rédacteur du Mercury, dont Philozoon c'est le pseudonyme de l'auteur - veut calmer (!) les inquiétudes par les vers suivants :

Un sot trouve toujours un plus sot qui l'admire

Et ses écrits,

Un Marchand pour les vendre et des sots pour les lire.

Ces six courts textes mettent en place des cadres polémiques dont on appréciera la rémanence: existence de stéréotypes nationaux - John Bull, le Yanké, les "amis du roi" (faux en l'occurrence) et Jean-Baptiste, qui passe à tort pour l'une des figures de "Sauvages" — dont l'argumentation et la fiction peuvent jouer ${ }^{21}$; ombre équivoque des États-Unis planant sur les relations entre Canadiens et Anglais et investie d'un pouvoir sur les choses -

20. Ce texte doit être placé en corrélation avec la publication ultérieure ( $\mathrm{n}^{\text {os }} 4-5$, 13 et 20 décembre 1806) de "Plaidoyers de la Cause en Appel entre Lavergne et le Curé et les Marguilliers de la paroisse de St. Léon Legrand [sic], et le Procureur du Roi intervenant pour Sa Majesté", où l'on voit que ces règles sont bafouées. Aucun commentaire ne le signale cependant.

21. Pour une analyse de ce jeu complexe sur la notion de "figures de la nation", voir Micheline Cambron, "Figures de la nation. De l'un et du multiple". Actes du colloque Canada, Identité 2000, tenu à Graz les 5-6 décembre 1999, à paraître. 
propriétaire de Museum, force météorologique — propre à rapprocher comme à séparer les Canadiens et les Anglais; déni de toute distinction entre les partis dans l'ordre constitutionnel "Les Anglois d'ici ne doivent pas plus avoir le titre d'Anglois que les Canadiens celui de François. Ne serons-nous jamais connus comme un Peuple , comme Américains Britanniques?»; irruption de l'image du mauvais Anglais désireux de créer une "aristocratie mercantile", plus pernicieux pour la nation que le Canadien, loyal malgré sa langue; évocation de l'histoire d'autres peuples pour soutenir l'image positive de la Constitution ${ }^{22}$. Mais on peut aussi y lire une représentation fort complexe de l'idée de révolution. D'un côté, Robespierre a "des yeux noirs et des sourcils fournis, et le sourire ambigu "; les Girouettes, ceux qui tournent et exécutent littéralement des "révolutions", sont dénoncées sans ambiguiité; la révolution américaine est dépréciée - il existe une "liberté Yenkée de la presse", d'une autre nature en apparence que la liberté de la presse ordinaire. De l'autre, la magnification de la liberté de la Presse, reprise en moult endroits, l'évocation de Tyran et de Catilina, honnis, paraissent justifier la révolution lorsque le bonheur du peuple est en jeu. D'ailleurs, cette idée qu'il existe une loi au-dessus des magistrats explicite également le postulat selon lequel le lien social répond à des règles précises qui transcendent les circonstances contingentes. Ce paradoxe rejoint en partie le récit originel du journal mais, outre la forme ambiguë qu'il imprime aux détails de l'argumentation, il permet de saisir que l'idée de révolution joue un rôle beaucoup plus central qu'il n'y paraît dans l'élaboration des discours.

Un survol rapide des premiers numéros confirme ce rôle. Ainsi, le deuxième numéro (29 novembre 1806) est à demi consacré à une lettre du Général Murray ${ }^{23}$ qui est un rapport sur "l'état présent de la Province de Québec [et] la nature des désordres qui y ont eu lieu". Outre que Murray y attaque violemment les Anglais occupant des responsabilités administratives et se plaint de la "taxe pesante" levée par Londres et de la "rapacité des gens de

22. Plus tard, au fur et à mesure que se produira l'éveil des nationalités en Europe, les conflits entre les visées impérialistes de certains états et le sort malheureux des petites nations vaincues (principalement la Pologne, l'Écosse, l'Irlande) confirmeront au contraire l'existence d'une oppression britannique et la nécessité d'un affranchissement.

23. "Traduction d'une LETTRE DU GÉNÉRAL MURRAY aux Lords du Commerce et des Plantations" [rédigée entre 1764 et 1766]. 
loi Anglois" (p. 6), cette lettre oppose les Canadiens aux "commerçants licencieux de New-York" et les loue pour leur soumission: "Ils obéirent de bon coeur dans l'espérance que leur bonne conduite seroit une recommendation pour eux à la faveur et à la protection de leur Souverain". Si l'on en croit Murray, bien qu'ayant, plus encore que les Américains sujets d'être mécontents, les Canadiens auraient rejeté l'idée de révolution. Cela ne semble pas être l'opinion d'Anglicanus, du Mercury, attaqué par Anglo-canadiensis, dont le texte anglais fut inséré dans le Mercury et la traduction reproduite dans ce même numéro du Canadien. Anglicanus considère plutôt les Canadiens comme un empêchement à l'érection du "rampart" "que nous devons élever contre l'ambition françoise, en répandant la langue Angloise", ils seraient donc des révolutionnaires par atavisme. AngloCanadensis retourne l'argument: "si suivant son raisonnement [celui d'Anglicanus] la langue Angloise est un rempart, contre l'ambition françoise, pourquoi la langue françoise n'en serait-elle pas un contre les spéculations américaines, surtout dans un tems où le Congrès, qui parle Anglois, dicte des loix de commerce à nos marchands britanniques". D'ailleurs, ajoute-t-il plus loin, citant Burke, "toutes les Colonies Protestantes se sont révoltées, elles se sont jointes à la France, et il est arrivé que le Canada Papiste a été le seul Pays qui soit demeuré fidelle, le seul sur lequel la France n'a pas mis le pied, la seule Colonie peuplée qui reste maintenant à la Grande-Bretagne". Le reste du numéro comporte une blague sur l'invasion américaine et quelques entrefilets: des nouvelles d'Europe, dont on s'attend à ce qu'elle entre dans une guerre générale (ce qui rend la menace napoléonienne évoquée par Anglicanus bien réelle ); l'annonce de la "séparation du territoire à l'Ouest des Alleghanis de l'union Américaine"; et enfin une fable dédiée au Mercury. Entremêlé aux attaques contre le Mercury se déploie tout un imaginaire de la révolution, marqué à la fois par les intentions impérialistes, mercantiles, des voisins du Sud et par celles, géopolitiques, de Napoléon. La révolution n'a ici rien de glorieux, elle est à l'origine "du débordement du torrent de l'ambition française [...auquel...] tous les amis de la liberté "doivent s'opposer, elle laisse sans frein les intentions spéculatrices des Américains.

Cette conception négative de la révolution trouve des échos dans un texte ironique publié dans l'édition du 6 décembre. Si les "révolutions des États politiques ne sont que des événements or- 
dinaires et l'effet nécessaire de l'instabilité des choses humaines", il est étonnant, expose l'auteur, Cunctaror, que les Dieux soient sujets aux révolutions. Or, c'est ce que révèlent les Annales du Temple de Mémoire ( $1^{\text {er }}$ décembre A.M. 5699). De nouveaux Demi-Dieux règnent sur les Arts, les sciences et les Belles-Lettres. Ceux-ci portent comme noms les pseudonymes utilisés dans le Mercury (Akritomuthos, Anglicanus, Touchstone, Amicus, Sancho Impromptu) et ce dernier (rebaptisé Mercure) "est créé le nouveau messager des Nouveaux Demi-Dieux". L'emploi hybride de la mythologie et de l'uchronie, est amusant, mais le trait essentiel de ce texte consiste à présenter les rédacteurs du Mercury comme étant sujets à la révolution, au retournement. Cela vise à les associer aux Américains, mais cela a aussi pour effet de ridiculiser la révolution, présentée comme un retournement factice puisque comme le conclut le texte: "Il n'est pas nécessaire d'observer que le Politique doit invoquer Akritomuthos, Le Jurisconsulte, Anglicanus etc. que le culte demeure le même, que la différence ne consiste que dans les nouveaux noms, et la nouveauté des Nouveaux Demi-Dieux". Le côté girouette de la révolution, qui rend l'opération moins glorieuse que ridicule, est perceptible également dans le désir du Canadien de recevoir un poème parodique sur l'Air de Yankee Doodle, chanson-phare de la révolution américaine ${ }^{24}$.

Si nous relisons ces morceaux pour en tirer une définition conséquente de la révolution, il faut remarquer que ce sont les "révolutionnaires" qui sont donnés pour des usurpateurs ou des tyrans en devenir. Le Régime anglais est donné pour légitime. Cela explique sans doute que la Conquête ne soit pas évoquée et que la capture de Buenos Ayres par l'armée Britannique soit connotée positivement - on énumérer les gains que la population trouve au changement de régime ${ }^{25}$. La République américaine est, au contraire, présentée comme agissant insidieusement pour gagner un espace commercial — c'est le vent de Nord-Ouest ${ }^{26}$ ou même ouvertement pour conquérir du territoire — c'est la déclaration officielle du Gouverneur du Vermont qui prétend rectifier

24. Curieusement, cette chanson avait d'abord été créée par les Britanniques pour se moquer des prétentions américaines. Les révolutionnaires américains s'en emparèrent retournant alors le sens de la chanson.

25. "Prise de Buenos Ayres", p. 4.

26. "No III" [Mr. Cary...], p. 2-3. 
les frontières que l'on trouve reproduite dans le numéro ${ }^{27}$. Les Anti-Canadiens sont les mêmes des deux côtés de la frontière américaine, ils sont au service de leurs intérêts mercantiles; ceux qui les ont combattus sont cités en exemple.

Certes, la conquête apparaît comme un moment de désordre et ce désordre correspond à n'en pas douter à notre révolution: le retournement politique s'effectue laborieusement suite à la dissolution du lien social antérieur — celui de l'Ancien Régime français - car le départ des élites, la désorganisation des liens hiérarchiques habituels, les aléas de l'histoire (dont l'occupation américaine) conduisirent vraisemblablement à une provisoire dissolution du droit. Selon les termes de la pensée de Locke, la dissolution du lien social est l'occasion pour le peuple de recouvrer sa souveraineté ${ }^{28}$. Or, comme l'écrit Jean Goulemot, s'il y a dissolution, "la puissance souveraine se trouve détruite dans sa forme institutionnelle et retourne à sa source première: le peuple réuni en communauté sociale mais non politique ${ }^{29}$ ". Ce retour fut d'autant plus facile que les aménagements de l'ancien Régime qui caractérisaient la Nouvelle-France confondaient les deux ordres, le régime seigneurial y ayant à la fois une dimension sociale et une dimension politique. L'émiettement des pouvoirs administratifs en place et le maintien du régime seigneurial, permettaient par leur cooccurrence que soit ramené le politique dans l'orbe des liens de proximités, avec au-dessus, mais sans relation véritable avec le reste, une intendance militaire anglaise. Cette période de dissolution se poursuivit jusqu'à l'Acte de Québec, premier acte législatif prenant en compte le caractère particulier de la nation, suivant le souhait de Burke ${ }^{30}$ et rétablissant la communication entre les ordres social, politique et législatif. Cet Acte marque l'entrée dans un nouvel ordre défini par la Constitution Britannique, dont les

27. "Ce qui suit est extrait du discours du Gouverneur de Vermont à la Législature de cet État, le 20 d'Octobre", p. 4.

28. Deuxième traité $d u$ Gouvernement, "De la dissolution du Gouvernement" et "De la conquête", dans John Locke, Deux traités de gouvernement, Paris, Vrin, p. 252-277 et 238-249.

29. Op. cit., p. 199.

30. Speech on Conciliation with the Colonies, dans Edmund Burke. On Government, Politics and Society, New-York, International Publication Service, 1976, p. 159-187. Burke parle, à propos des Colonies américaines, de "temper and character" (p. 171). Burke est souvent cité dans Le Canadien. Comme Charles James Fox, il fait figure à la foi de protecteur du fragile statut colonial et d'autorité morale. 
termes s'appliquent désormais aux nouveaux sujets: si l'on postule que le peuple canadien s'y donne volontairement, il faut y voir en quelque sorte une révolution d'instauration ${ }^{31}$. Comme ce nouvel ordre est présenté comme répondant aux voux de la population canadienne dans le journal Le Canadien, cela permet d'indexer les opposants à l'établissement de ce lien du côté des tyrans et aussi de suggérer que l'action des Américains relève de l'usurpation. La pensée de Locke joue ainsi un rôle séminal dans la mise en forme de la représentation du politique qui s'exprime puisqu'elle autorise aux yeux des rédacteurs du Canadien, un partage entre les tenants de la légitimité et ceux qui la bafouent, les Anti-Canadiens. Ainsi, ce n'est pas un hasard si le texte qui commente les santés anticonstitutionnelles des opposants au bill des Prisons ${ }^{32}$ se conclut sur l'évocation de tyrans. Locke, dans son chapitre "De la tyrannie 33 ", opère la même association: l'évocation des tyrans de l'histoire est accompagnée d'un commentaire sur le franchissement des bornes reconnues à l'autorité, "Là où le droit finit la tyrannie commence ${ }^{34}$ ".

\section{Les archives et l'histoire}

C'est à cet aspect de l'argumentation que la citation de pièces d'archive contribue le plus fortement. En effet, divers documents sont reproduits dans les pages du journal, le plus souvent sans commentaires immédiats, qui tendent tous à "faire l'histoire du parti anti-Canadien", à montrer l'action anticonstitutionnelle de ceux qui s'opposèrent ou s'opposent encore à l'existence d'une égalité constitutionnelle entre les "anciens" et les "nouveaux" sujets. Ces diverses pièces d'archive constituent autant d'arguments qui visent à prouver l'existence d'un parti anti-Canadien, la bienveillance des autorités coloniales et la fidélité des Canadiens à la Constitution britannique. Elles occupent une partie considérable de la surface du journal et, structurellement, elles sont ensuite littéralement citées dans des argumentations diverses, que celle-ci émane de la rédaction ou de ses opposants. Dans les neuf premiers numéros, on peut voir reproduits les documents suivants -

31. Il s'agit ici de l'instauration du lien social; voir John Locke, op. cit.

32. Sur "la Querelle des prisons", voir Jean-Pierre Wallot, op. cit., p. 60-71.

33. John Locke, op. cit., p. 251-257.

34. Ibid., p. 252. 
dont certains sont traduits pour le bénéfice des lecteurs: "Lettre du Général Murray" (n 2, 29 novembre 1806); "Extraits des Débats du Parlement de la Grande Bretagne" ( $n^{\circ}$ 3, 6 décembre 1806); "Plaidoyers de la Cause en Appel entre Lavergne et le Curé et les Marguilliers de la paroisse de St. Léon Legrand [sic], et le Procureur du Roi intervenant pour Sa Majesté" (publiés sur deux numéros, 4 et 5, 13 et 20 décembre 1806); "Extrait des débats du 15 Mai 1776 de la Chambre des Lords sur la pétition des habitants britanniques de la Province de Québec", "Extrait de l'Adresse du Congrès au Peuple de la Grande Bretagne en 1774", "Extrait de l'adresse du même Congrès aux habitants de la Province de Québec du 26 octobre 1774", "Extrait de la Pétition du même Congrès au Roi en 1774", "Extrait de Hatfell's Precedents vol. 2 page 94" ( $n^{\circ}$ 6, 27 décembre 1806); "Extrait du Journal de la Chambre d'assemblé. Mardi 1er avril 1806" ( $\mathrm{n}^{\circ} 7$, le 3 janvier 1807); "Extrait du Journal de la Chambre d'assemblée. Vendredi 22e. Février 1805", "Mémoire contenant les motifs [...] ${ }^{35}$ " (nº 9, 17 janvier); pièces auxquelles il faut ajouter, pour faire bonne mesure, les témoignages sur la guerre américaine, les extraits de textes du Mercury qui sont reproduits ${ }^{36}$, et les notices nécrologiques, dans lesquelles on rappelle souvent des faits d'armes. Ces documents sont complétés par des assertions données soit comme des nouvelles, soit intégrées à des lettres de lecteurs. Ce qui est en jeu c'est, bien sûr, la véracité des faits allégués dont les documents portent témoignage - ils informent, rectifient, confirment - mais aussi la publicité offerte à des discours qui autrement échapperaient à la connaissance commune. Certains en appellent même à une guerre d'archives:

Nous voudrions bien savoir de ces Messieurs, pourquoi de si bonnes gens qu'eux ont toujours eu le malheur d'être mal avec tous les gouverneurs. Leurs amis de l'autre côté pourroient peut

35. Il s'agit d'un mémoire portant sur le mode de taxation retenu pour l'érection des prisons de Québec et de Montréal. Voir supra, note 32.

36. Par exemple l'entrefilet suivant: "PLAN ACTUEL des Anti-Canadiens extrait du discours d'AKRITOMUTOS, $\mathrm{n}^{\circ} 47$ du Mercure", où l'on trouve le couplet sur l'anglification: "Que reste-t-il à faire? à retrancher ces privilèges qui sont regardés comme peu considérables, mais qui le sont trop en effet, et dont les conquis usent trop librement; à avoir l'administration publique des affaires traitée en Anglois seulement, par des Anglois ou des personnes de principes Anglois; ce qui est le premier pas et le plus important à faire pour parvenir à Anglifier la Province, changement qu'on doit ardemment s'efforcer d'obtenir". 
être avoir dans leurs archives des pièces qui pourroient les justifier sur ce point ${ }^{37}$.

S'il y a, en sous-main, l'idée d'un véritable complot anti-Canadien lisible dans ces traces éparses, on peut aussi y voir la construction d'un espace commun du discours qui pourrait à la fois accueillir les défenseurs des Canadiens et ceux qui parmi les anciens sujets savent se libérer de leurs préjugés. Le souci de faire prévaloir la liberté de presse transforme ainsi le journal en forum où l'on débat autour de pièces diverses convoquées pour étayer des argumentations qui demeurent parfois implicites - ce qui oblige parfois la rédaction à justifier la reproduction de pièces d'archives et à faire des mises au point quant à ses intentions ${ }^{38}$.

Il est à ce titre frappant de voir que le journal accueille des lettres de lecteurs qui s'opposent à sa politique éditoriale (on entretient de grossiers préjugés, expose plaisamment Démocrite $\left(n^{\circ} 5\right)$, on ne parle pas assez d'agriculture et trop des fautes passées des Anglais, se plaint D. ( $\left.\mathrm{n}^{\circ} 8\right)$, vos sources sont mauvaises, explique avec humeur De Lanaudière. Celui-ci est le seul à ne pas signer d'un pseudonyme. Il faut noter cependant que D. avait lui aussi signé de son vrai nom, c'est la rédaction qui lui attribue un pseudonyme; elle s'en explique de la manière suivante:

Tout ce qui paroît dans un papier de la nature de celui-ci doit se soutenir de lui-même par la notoriété des faits et l'évidence des raisons, et ce seroit introduire un vrai charlatanisme que de vouloir y faire ajouter foi par d'autres moyens

Mais ce qui doit frapper davantage, c'est que cette méthode de publier les écrits avec de vrais noms convertiroit sur le champ la presse la plus libre en un véhicule de flatterie [...] Ce seroit une presse que l'état le plus despotique pourroit admettre sans crainte, au reste nous ne pensons pas que l'auteur de ce texte ait eu la moindre idée de donner une pareille tournure à la nôtre ${ }^{39}$.

37. Ce commentaire fait suite à un article où l'on rapporte que le Mercury a donné des extraits de six lettres "d'Anti-Canadiens des États-Unis" témoignant de l'aide reçue de Canadiens en 1775, au moment de l'Invasion américaine. Le Canadien commente l'accès libre que les Anti-Canadiens des deux côtés de la frontière semblent avoir de leurs archives respectives. Le samedi 10 janvier 1807, p. 28.

38. Par exemple, à propos de la publication de la lettre de Murray, la rédaction se défend d'avoir voulu induire que les marchands actuels soient de la même racaille que celle qui arriva lors de la Conquête ( $\mathrm{n}^{\circ}$ 4, 13 décembre 1806, p. 15-16).

39. $\mathrm{N}^{\circ} 8,10$ janvier 1807 , p. 28. 
Les discours et les pièces d'archives sont véritablement offerts au jugement du lecteur et leur signification est donnée "par la notoriété des faits et l'évidence des raisons". Le consensus social devrait donc se construire "naturellement" par la diffusion des informations pertinentes.

Les refigurations des deux récits que nous avons lus dans le Prospectus se trouvent donc simultanément nourries par le recours à l'archive puisque celui-ci explicite le récit de rupture, celui qui doit être effacé par la publicité que permet la liberté de la Presse qui abat le préjugé, et, dans le même temps repose sur le postulat que tout homme de bonne volonté est nécessairement entraîné à induire des mêmes pièces d'archives, les mêmes raisonnements.

Dans cette perspective argumentative et narrative complexe, l'historiographie est donnée pour un savoir "naturel", si tant est que la totalité des pièces soit rendue publique, et l'histoire ellemême comme porteuse de leçons. Le respect de la loi et de la coutume, la bienveillance des Gouverneurs britanniques du Parlement et de Sa Majesté dont rend compte l'Acte de Québec qui "accorde à tous les Sujets Canadiens de Sa Majesté leurs anciennes coutumes et usages et la jouissance du libre exercice de la Religion Romaine ${ }^{40}$ " et la connaissance des règles de l'argumentation permettent juger de chacune des pièces convoquées, de déceler le sens de l'histoire, c'est-à-dire ce vers quoi elle tend: un pacte social qui prenne en compte toutes les parties de la population du Canada. Cette dimension "naturelle" de l'histoire et du jugement historiographique est particulièrement perceptible lorsque l'on cherche à comprendre le sens que pouvait revêtir pour les rédacteurs du Canadien la reproduction des "Plaidoyers de la Cause en Appel entre Lavergne et le Curé et les Marguilliers de la paroisse de St. Léon Legrand [sic], et le Procureur du Roi intervenant pour Sa Majesté». Celle-ci ne s'explique que parce que les termes du jugement sont à la fois contraires au bon sens - la paroisse en question n'existe pas, car il n'y a pas de document qui

40. Samedi, 27 décembre 1806, p. 11. Je ne restitue pas les italiques disposés çà et là, qui ne sont peut-être pas significatifs, le texte étant placé à la toute fin du numéro et réduisant la notice d'imprimerie. Or nous savons que le journal ne dispose que de peu de caractères (il renonce à allonger la parution et signale attendre un nouveau jeu); il se pourrait que ces italiques soient donc une solution de fortune pour pallier le manque de caractères. 
prouve son existence (!) - et un déni des termes mêmes de l'Acte de Québec. En fait, si l'on suit le raisonnement du journal, les Anti-Canadiens sont de mauvaise foi - ils sont avides d'usurper un pouvoir que la Constitution ne leur reconnait pas - et ils manquent de sens commun ${ }^{41}$.

\section{Poésie et sens commun}

Cette dimension est perceptible dans les textes plus proprement littéraires publiés par Le Canadien durant cette période: une fable, quatre "épigrammes ${ }^{42}$ ", trois chansons, un poème et une Étrenne du Gazetier (publiée sur une feuille à jeter), lesquels éclairent à la fois l'enjeu central du débat, l'assimilation par l'anglification de la population canadienne et le ton sur lequel est traité ce grave sujet. Contrairement au reste du journal, ils ne se présentent pas comme des documents susceptibles de concourir à écrire l'histoire du passé ou celle de l'avenir: ils sont au contraire en porte-à-faux puisqu'ils dénoncent ou fictionnalisent explicitement la situation du moment et tendent à ridiculiser l'ennemi : les Anti-Canadiens et le Mercury.

La fable et les épigrammes, visent à dénoncer la hargne gratuite des adversaires, comparés à la ronce rampante qui déchire par plaisir les passants (p. 8); au carosse de Voiture — "il faut qu'il parte, vide ou plein" (p. 12); aux effets néfastes du mercure - il "Ne fait qu'étourdir la tête,/Et nous faire mal au cœur" (p. 16); au moissonneur (l'Anglais) qui passe le premier dans un champ et ne laisse à l'autre (le Canadien) qui le suit et travaille autant le seul profit de glaner ("Les Moissonneurs", p. 20). Les chansons reprennent en l'amplifiant la même thématique. Mais leur forme même et l'utilisation, glosée, d'un timbre dont la fonction comique est ici évidente, Yankee Doodle, donnent une ampleur nouvelle à l'ironie. La première Chanson, sur l'air enlevé de Robin ture lure lure, se moque de l'Apothicaire Racy (c'est le nom de l'imprimeur du Mercury), qui vend son Mercure, "cette drogue

41. Notons au passage que c'est l'un des sens que peut prendre, par extension, le mot girouette.

42. Il s'agit de textes ayant une forme épigrammatique plutôt que d'épigrammes au sens fort. L'un des textes porte sur Napoléon ("L'amitié déplacée", p. 32). C'est le seul morceau littéraire qui ne porte pas explicitement sur la polémique à l'origine de la naissance du journal. 
130

bonne à rien": "Bon et sage Canadien /N'a point besoin de Mercure". On suggère alors à l'apothicaire d' "aller chez nos voisins" (p. 16). Tout est là : le refus des Canadiens de la médecine du Mercure, l'insinuation des services rendus aux Américains par le journal ennemi, la moquerie contre le commerçant qui connaît mal son marché. L'auteur du texte est invité par la rédaction à commettre une autre chanson, cette fois sur l'air de Yankee Doodle, "afin de nous exercer à l'avance", puisque nous allons être obligés de nous anglifier suivant la Méthode d'Anglicanus et cie" (p. 16). L'auteur décline (le 27 décembre p. 24). Est-ce lui qui écrit l'étrenne du $1^{\text {er }}$ janvier 1807? Étroitement attaché au thème du Mercury-Apothicaire, ces Etrennes du Canadien ${ }^{43}$, sur l'air Le Saint craignant de pécher (ou C'est notre bon père Noé), airs qui évoquent des chansons comiques qui portent sur des personnages ridicules à force de sottise, composent à la fois un condensé de la prose du Mercury - et du Canadien - et une attaque contre le peu de jugeotte de ceux qui croient défranciser à si peu de frais la population canadienne.

Un bref couplet d'introduction, chanté par un "je" qui est celui du petit gazetier, pose le cadre pragmatique propre à l'étrenne:

Je veux vous complimenter

Mais comment donc faire?

S'il faut me défranciser

Pour pouvoir vous plaire;

Le gazetier veut et doit offrir ses souhaits, qui lui vaudront en retour quelque obole, mais il ne sait comment se "défranciser" (unfrenchify). Il énumérera ensuite les remèdes proposés par le Mercury, mêlant plaisamment les termes de médecine - ancienne, pour nous - et les discours du Mercury pour conclure à l'échec de tous les "spécifiques" proposés car:

Il manque à tous vos essais,

Imprudents chimistes;

Un scrupule de Rablais

Pris par improviste, et dix onces de raison,

Ajoutez y un poinçon

43. Voir l'annexe. Ce poème a été reproduit avec d'éclairantes notes explicatives dans Yolande Grisé et Jeanne d'Arc Lortie, Les textes poétiques du Canada français 1606-1867, vol. 2, Montréal, Fides, 1987, p. 25-27. Il est aussi reproduit, avec la partition, dans Maurice Carrier et Monique Vachon, Chansons politiques du Québec, t. 1, Montréal, Leméac, 1977, p. 116-117. 
De bon sens d'emprunt,

De cervelle un pot,

Du commun,

Au plutot

Par force électrique

Ou par émétique.

Pour ceux qui n'ont pas lu les précédents numéros du Canadien, le texte paraît allégorique et obscur ${ }^{44}$. Mais les personnages dont on se moque correspondent aux pseudonymes qui signent les textes du Mercury - Gentleman Trader, Akritomutos, Nestor, Racy, Touchstone - et les thèmes de chacune des strophes évoquent un "spécifique" qui rappelle la teneur des articles du Canadien: "l'esprit de poltron bien clair" ironise sur la poltronnerie des Anglais ${ }^{45}$; "l'huile de semideos" ridiculise les signataires des articles du Mercury, ces "Nouveaux Demi-Dieux" qui ont fait une fausse révolution (p. 12); "la cire en astringent à la Yankee Doodle" rappelle les pointes à l'égard des Américains et les discussions autour des autres chansons publiées par Le Canadien; la dernière mixture comporte l'air des sept girouettes (p. 3-4), du Castoreum, de l'Amicus Syrop et de l'Apéritif Sancho, avec "dragme d'impromptus/De Touchstone un gros", bref de la bouffonnerie et du commerce. Ces remèdes cocasses sont directement attachés à certains des discours du Mercury, celui-ci apparaît alors non seulement comme un assemblage d'"Imprudents chimistes", mais surtout comme un charlatan (dans la tradition des apothicaires molièresques), un usurpateur.

Démophon va quant à lui composer la chanson sur l'air de Yankee Doodle que la rédaction réclamait: il la date du $1^{\mathrm{er}}$ janvier mais, moins adroit que l'auteur d'Étrennes, il développe surtout l'idée que c'est chez les "Yankies" que le Mercury désire que l'on aille apprendre la langue, puis la politique. En définitive, "Yankee Doodle on chantera, / Après quoi on s'écriera: / Vive la République». Rien que de très conforme donc à l'argumentation du $\mathrm{Ca}$ nadien. Le contraste est net, dans la seconde chanson, Démophon polémique sérieusement alors que dans Étrennes, l'auteur

44. C'est l'avis tout à fait justifiable de Yolande Grisé et Jeanne d'Arc Lortie, op. cit., p. 26.

45. Il est constamment question de la prétendue poltronnerie des Canadiens, que les faits d'armes racontés dans les rubriques nécrologiques dénient; et de la poltronnerie des Anglais, qui ne se bousculèrent pas comme volontaires lors de l'Invasion américaine. 
prend ses distances et s'amuse, bien au-dessus de la mêlée. Un dernier poème enfin, "Hymne des bons Canadiens - À LA PAIX / Pour Janvier 1807", reprend assez platement la topique des bienfaits de l'Angleterre et appelle la Paix à demeurer "sur ces bords": "ne t'en éloigne jamais". Il faut souligner ici que les meilleurs textes, ceux qui présentent un intérêt esthétique, sont ceux qui jouent le mieux de l'absurde que crée la distance.

Cette distance est clairement l'enjeu du poème "La liberté de la presse" ( $n^{\circ} 6$, p. 14), qui loin des grandes envolées philosophiques du journal, en fait un sujet bouffon où ignorants et sots se trouvent ridiculisés :

Certain rustaut aussi malin que bête,

Fendoit la presse et pour percer les rangs,

Heurtoit, poussoit et coudoioit les gens ;

Quelqu'un lui dit: l'ami, un peu moins de rudesse,

Vous nous frottez fort inutilement,

D'ici on peut ouir la messe;

Moi-même je n'ai pu pénétrer plus avant.

Mais fine, repart l'habitant,

Tant pis pour vous si ça vous presse;

Qué qui v'z'empêche d'en faire autant,

J'n'avons ty pas la liberté d'la presse?

La bouffonnerie rappelle aussi que des libertés aussi essentielles demeurent trop souvent des mots vides de sens. Bref le récit idéal, celui de la réconciliation nationale grâce à la connaissance mutuelle se heurte au manque de raison de certains citoyens. Lus dans cette perspective, ce poème et Étrennes constituent de solides mises en échec du postulat de la raison "naturelle". Eux seuls échappent à la polarisation des récits, qui se trouvent renvoyés dos à dos: les prétentions du Mercury - et le retournement d'icelui par Le Canadien - manquent de raison et le récit de l'harmonie constitutionnelle est illusoire, car le sens commun ne saurait régner.

Le rôle curieux qu'assume alors la littérature mérite d'être rapproché de la forme du même du journal. Revenons quelque peu sur l'étrenne. Elle est diffusée en dehors du journal, dont elle reprend par ailleurs de façon microcosmique l'ensemble du discours: elle est un double externe, mais un double sans illusion, que la distance ironique empêche de succomber à l'illusion de l'harmonie du monde que le journal semble porter. Ce double est oral, fait pour être chanté — c'est aussi le cas de la chanson sur 
la liberté de la presse, incompréhensible en dehors de son oralisation - et il attaque un journal, un papier qui est donné allégoriquement comme une pharmacopée absurde dont les effets s'apparentent plus à ceux de la vaccine qu'à une guérison - qui en l'espèce serait la "défrancisation ${ }^{46}$ ". Entre les récits en présence, celui des déboires récents liés à l'aveuglement des AntiCanadiens, stratégiquement mis en marge de la société, et celui de l'harmonie fondée sur l'exercice de la raison "naturelle" rendu possible par la "communication continuelle ${ }^{47}$ " dont le journal est le support matériel, Étrennes - et dans, une moindre mesure, les autres textes littéraires - imagine un discours qui reconduit le pouvoir du journal dont il est le microcosme tout en sapant ses assises utopiques.

Comme le rappelle fort justement Marshall McLuhan, le passage de l'oralité à l'écriture fut, dans la Grèce ancienne, une révolution déterminante qui provoqua la réflexion forte et riche d'un Platon, mais la révolution qui se joua au tournant du dixneuvième siècle, lorsqu'on passa d'une société du livre à une société du journal — c'est-à-dire de l'exercice privé de la lecture à son exercice dans l'espace public et du déploiement linéaire d'un texte à la juxtaposition dans un même espace de plusieurs textes - ne fut pas moins radicale ${ }^{48}$. Pour les Canadiens, ce changement coïncide avec l'émergence de récits qui sont des récits de la nation: ceux-ci, rappelons-le ne préexistaient pas à leur déploiement dans les journaux. Le premier récit, celui de l'harmonie, est explicitement raconté dans les professions de foi dans la raison "naturelle", il organise syntagmatiquement les termes de la Loi en une seule narration. Le second, en voie d'ordonnancement, est le récit du parti anti-Canadien, recomposé par bribes grâce aux diverses anecdotes que le journal rapporte par l'intermédiaire des pièces d'archive convoquées. Ce récit est signe de la

46. Il est tentant de rapprocher l'étrenne du Canadien du Phèdre de Platon, là où l'écriture est à la fois remède et poison.

47. Prospectus.

48. Dans Hot and Cold, Toronto, Signet Book, 1967, p. 128-142, McLuhan examine les transformations induites dans l'esthétique savante (chez Lamartine, Mallarmé et Joyce, entre autres) par la forme même du journal: "It is strange that the popular press as an art form has often attracted the enthousiastic attention of poets and aesthetes while rousing the gloomiest apprehensions in the academic mind." Certaines des remarques qui suivent sont inspirées de cette analyse qui m'a aimablement été signalée par Gilles Marcotte. 
134

nécessité de redonner sa forme originelle au pacte social - ce qui prend bien sûr une forme antithétique dans le journal d'en face, le Mercury, qui use cependant des mêmes modalités de construction. Or, ce second récit ne peut prendre forme, c'est-à-dire passer de l'état de pièces éparses à celui d'histoire, que dans l'espace du journal. Le premier donne une plus grande impression de cohérence, car la Loi paraît une: bien que ce soit illusoire, sa cohérence narrative, qui tient à sa projection dans une société idéale, semble lui venir d'au-delà d'elle-même, de ces Lois "naturelles" qui impriment si fortement leur marque sur la philosophie des Lumières. Mais là encore la diffusion de ces postulats revient au journal, qui ne manque pas d'ailleurs de rappeler que les fondements de son action tiennent à la grandeur de l'exercice de la raison. Ces deux récits, qui sont encore aujourd'hui nos fictions fondatrices, ont un pouvoir politique rémanent qu'il faudra bien un jour percer. L'existence de telles fictions n'est évidemment pas propre à la société canadienne - et québécoise. Mais, et peut-être est-ce là une esquisse de réponse, la coïncidence de leur émergence et de la mise forme de l'espace public rend indiscernables les frontières entre ces fictions et la configuration de l'espace public. Les formes de cet espace public commençant tout juste à changer avec l'avènement des médias électroniques, il n'est peut-être pas étonnant que nous soyons restés enfermés tout ce temps dans nos récits fondateurs, sans cesse redéployés et confirmés dans la forme même de notre espace public. Il n'est pas dit toutefois que nous puissions faire l'économie de ces récits. Leur disparition, dans la foulée d'une mondialisation qui tend à dissoudre l'espace public dans un mercantilisme radical des échanges, risque d'effacer le politique même, et avec lui toute idée de lien social. 